\title{
Información técnica para el desarrollo agropecuario y forestal del estado de Yucatán
}

DOI: 10.22403/UQROOMX/TYP04/09

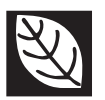

Francisco Bautista-Zúñiga*

\section{Resumen}

La precaria situación de la agricultura del estado de Yucatán, aunada a la marginación de las comunidades rurales intensifica la insuficiencia alimentaria (desnutrición); la emigración de los varones en busca de trabajo; la desintegración familiar y contribuye a la pérdida de identidad, entre otros problemas de salud y sociales. Los tomadores de decisiones que participan en la administración del espacio físico de Yucatán, así como los productores agrícolas, forestales y pecuarios, no han contado con información sufi-cente para fundamentar sus resoluciones. Esta situación ocasiona la degradación de los recursos naturales, el fracaso de planes agrícolas, la fuga de capitales por compra de alimento y desempleo, entre otros problemas. En este artículo se propone la creación de un Centro de Información Ecológica, Ambiental, Agropecuaria y Forestal, con bases de datos georeferenciadas, que proporcionen información para la formulación de una política agrícola acertada y la planificación del uso de las tierras.

Palabras Yucatán, Centro de Información Ecológica, agricultura, bases

CLAVE de datos, planificación.

*Centro de Investigación en Geografía Ambiental, unAM / leptosol@yahoo.com 


\section{Breve diagnóstico de la situación agrícola actual en Yucatán}

El monocultivo del henequén (Agave fourcroydes) y la ganadería extensiva basada en el monocultivo de pastos han tenido un impacto negativo en la conservación de los recursos naturales del estado de Yucatán.

El uso de suelo para el cultivo de henequén es reemplazado por la producción de maíz con el sistema tradicional de roza, tumba y quema (RTQ). El maíz alcanza una producción de $1.5 \mathrm{t} \mathrm{ha}{ }^{-1}$ con fertilizantes y $0.7 \mathrm{t} \mathrm{ha}^{-1}$ sin fertilizantes en el primer año, $y$ de 0.75 a $0.5 \mathrm{t} \mathrm{ha}^{-1}$ en el segundo, lo que resulta insuficiente para el autoconsumo de las familias campesinas; además, el manejo agrícola de los escasos montes altos y medianos ejerce una mayor presión sobre la vegetación, fauna silvestre y suelos de la selva baja caducifolia.

En diversos foros, los ganaderos del estado han manifestado que en los pastizales se invierte una gran cantidad de esfuerzo y recursos económicos en el control de las arvenses, así como en la fertilización y riego. Estudios preliminares revelan que, a medida que avanza la degradación del suelo (compactación y pérdida de la fertilidad) la inversión es mayor. La degradación de los pastizales sólo se resuelve temporalmente con su abandono y con la apertura de nuevas tierras.

Por otra parte, el gobierno del estado ha intensificado la prevención de los incendios forestales al regular el uso de la quema en la agricultura, pero esta medida aumentará el costo en el control de las arvenses, tanto a los productores milperos como a los ganaderos, por lo que se requiere generar opciones para cumplir los objetivos del uso del fuego en la agricultura.

Ambos problemas (degradación del suelo y control de arvenses) ocasionan que agricultores y ganaderos tengan la necesidad de abrir nuevas áreas de vegetación para cultivar milpas o pastos, según el caso, lo que a su vez provoca una fuerte presión sobre los recursos de la selva y el suelo.

El elevado costo del mantenimiento de los sistemas monoespecíficos también los hace altamente dependientes de insumos y del mercado. Es por esto que, para seguir utilizando los henequenales, milpa de RTQ y pastizales, así como para el desarrollo de nuevos cultivos, se requiere mejorar los suelos, disminuir los costos de su conservación y estabilidad en el mercado. 
La precaria situación de la agricultura del estado de Yucatán, aunada a la marginación de las comunidades rurales intensifica la insuficiencia alimentaria (desnutrición); la emigración de los varones en busca de trabajo; la desintegración familiar, y contribuye a la pérdida de identidad, entre otros problemas de salud y sociales.

Los tomadores de decisiones que participan en la administración del espacio físico del estado, así como los productores agrícolas, forestales y pecuarios, no han contado con información suficiente tanto en cantidad como en calidad, que les permita tener los elementos de juicio para fundamentar sus resoluciones. Esta situación ocasiona la degradación de los recursos naturales, el fracaso de planes agrícolas, la fuga de capitales por compra de alimento y desempleo, entre otros problemas.

Las riquezas culturales y ecológicas han hecho del turismo una de las actividades principales del estado de Yucatán, en la cual se invierten grandes cantidades del erario para su fortalecimiento y promoción. Pero una forma de mejorar e impulsar el turismo es mediante la creación de condiciones de estabilidad y seguridad social, y el fortalecimiento de la identidad cultural; esto propicia una mejor y más amplia educación y promueve la generación de empleo bien remunerado, entre otros beneficios. La agricultura es una actividad de alta demanda de mano de obra y de importancia estratégica para la seguridad nacional (independencia alimentaria), por ambos motivos la mayoría de los países, por no decir que todos, subsidian la agricultura de muy diversas formas.

La lamentable situación agrícola, los problemas sociales de las comunidades rurales y el fortalecimiento de las actividades turísticas (mediante la conservación de las riquezas culturales y ecológicas) requieren, entre otras cosas, de un plan agrícola estatal de largo plazo.

\section{La información como base para la planificación y la toma de decisiones en el ámbito agrícola}

La capacidad de producción de las tierras viene determinada por el clima, las condiciones del suelo, su fisiografía, la calidad del agua y el nivel de la tecnología aplicada. Un manejo sostenible requiere de políticas correctas y una planificación basada en el conocimiento de estos recursos, sus requerimientos y las 
interacciones entre las tierras. Las respuestas a las cuestiones que se relacionan a continuación proporcionan las bases para la formulación de una política agrícola acertada y para la planificación del uso de las tierras.

- En función de sus aptitudes y limitaciones de uso, ¿cómo se distribuyen las tierras en el estado?

- ¿Qué usos se pueden recomendar sobre diferentes tipos de tierras en las diferentes localidades?

- ¿Cuáles son las zonas de mayor potencial agrícola, pecuario y forestal?

- ¿Cuáles son las tierras no aptas para las actividades agropecuarias?

- ¿Es adecuado el espacio asignado a las áreas protegidas?

- ¿Cuáles son las zonas de mayor riesgo de contaminación del acuífero por las actividades agropecuarias y forestales?

- ¿Cuáles son los sistemas agropecuarios y forestales más adecuados para los climas y suelos del estado?

- ¿Cómo podrían mejorarse las condiciones para un buen manejo de los sistemas agropecuarios y forestales?

- ¿Cuál es el balance entre las demandas de la población y la disponibilidad de tierras en áreas especificas y cómo responde este balance a las mejoras en las inversiones o el manejo?

- ¿Qué innovación y transferencia de tecnología son acordes a los ambientes del estado?

- Los mecanismos de comercialización de los productos, ison los adecuados?

Responder a estas preguntas permitirá el desarrollo de mejores políticas de uso de tierras y la creación de estrategias de desarrollo agropecuario y forestal que cuiden del ambiente y la biodiversidad, tales como: a) Información de fácil acceso y recomendaciones en áreas específicas; b) Suministro de insumos agrícolas; c) Establecimiento de prioridades en la investigación agrícola, y de redes para la innovación y transferencia agrotecnológica; d) Formulación de la legislación o líneas maestras que regulen y minimicen el impacto ambiental, y el establecimiento de un control medioambiental, y e) Identificación de programas o proyectos de desarrollo local. 
La promoción y apoyo a las actividades agrícolas sustentables dará como resultado: a) Generación de empleo de las comunidades rurales; b) Se reactivará la economía al evitar la fuga de capital por la compra de alimentos fuera del estado; c) El cuidado de los ecosistemas, que son una de las riqueza de la entidad.Todo esto, sin duda, redundará en el mejoramiento de la calidad de vida de la población del estado de Yucatán.

Por los problemas mencionados, se hace necesaria la creación de un Centro de Información Ecológica, Ambiental, Agropecuaria y Forestal del estado de Yucatán con bases de datos georeferenciadas que incluya los siguientes temas:

- Ecosistemas. Por la biodiversidad y servicios ambientales.

- Geoformas. Por los riesgos y amenazas ambientales, así como las restricciones y oportunidades agropecuarias y forestales.

- Suelos. Por las restricciones y oportunidades de uso agropecuario, forestal y urbano así como por los servicios ambientales.

- Climas. Para el manejo agropecuario y forestal así como por las amenazas meteorológicas.

- Acuíferos. Por la calidad agrícola del agua y las oportunidades y riesgos de uso.

- Cultivos. Producción animal y proyectos forestales (sanidad animal y vegetal, producción, etcétera).

- Tecnologías apropiadas a cada zona agroecológica.

Esta información, con un enfoque interpretativo, utilitario y práctico, además de taxonómico; con su evaluación, integración y modelación para la creación de escenarios mediante el uso de sistemas de información geográfica, será de mucha utilidad en investigación, docencia, transferencia de tecnología y divulgación sobre la importancia del manejo de los recursos naturales, tal como se hace en otros países; pero, principalmente, será muy útil para la toma de decisiones sobre el manejo de los recursos naturales del estado de Yucatán.

El centro de información permitiría, además, una mejor y más útil experimentación agrícola en beneficio directo de la producción agropecuaria y forestal; la transferencia de tecnología y su manejo tendrá mayores 
posibilidades de éxito. En cuestiones prácticas, los productores podrán contar con una gran cantidad de información técnica que sirva de soporte a la toma de decisiones a diversos niveles y escalas. El centro de información servirá también de apoyo en la formación de los estudiantes de todos los niveles educativos, y los grupos sociales preocupados por la salud ambiental y de los ecosistemas contarían con información técnica confiable.

\section{La operación del centro de información}

Las bases de datos de información ecológica, ambiental, agrícola, pecuaria, forestal y de infraestructura, por ejemplo: relieve, límites municipales, vías de acceso, cuerpos de agua, forma del terreno, suelo, áreas irrigables, uso de suelo, población, etc. se enlazarían e integrarían a un sistema de información geográfica para una consulta rápida y precisa. También podrían ser utilizadas para generar modelos del potencial de la tierra según los diferentes tipos de uso, así como modelos propios para la toma de decisiones en diversos ámbitos y por localidades.

Con esta información se producirían mapas de geoformas, suelos, climas, calidad y profundidad del acuífero, uso de suelo y vegetación, infraestructura agropecuaria y forestal, áreas naturales protegidas, así como contenidos acerca de cultivos anuales y perennes, dosis de fertilización por zonas agroecológicas, tipos de manejo agropecuario y forestal, especies endémicas, especies con algún grado de protección de acuerdo con las NOM y mapas de escenarios según aptitud de uso, entre otros.

Para la divulgación de la información, se traduciría la información científica, técnica y taxonómica a información de fácil comprensión para las diversas audiencias: niños y niñas de educación básica, productores campesinos, grandes productores, estudiantes universitarios y posgraduados, técnicos, autoridades, entre otros.

Serían necesarias instalaciones adecuadas al propósito del centro de información: una sala de exposiciones; un auditorio para conferencias y presentación de material audiovisual; sala de juntas; un taller para los trabajos del mantenimiento de las colecciones; una mapoteca para el cuidado del material cartográfico; un laboratorio básico para la realización de análisis rápidos de diagnóstico y demostrativos; una biblioteca (figura I y cuadro I). 


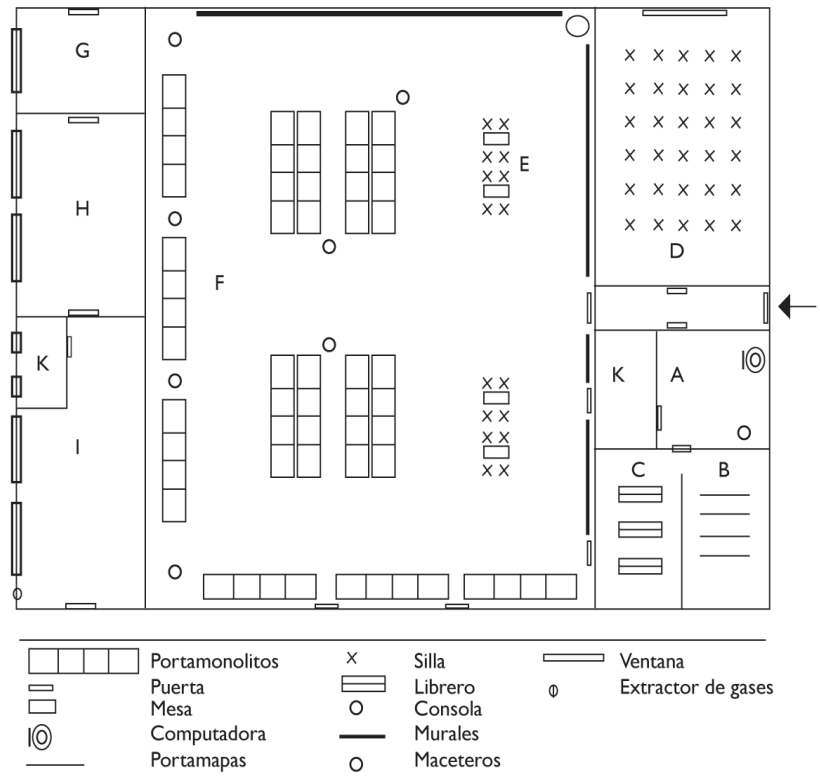

Figura I. Diseño de las instalaciones de un Centro de Información Ecológica, Ambiental, Agropecuaria y Forestal. A) oficina; B) mapoteca, C) minibiblioteca, D) sala de conferencias, E) sala de consultas, F) sala de exposiciones, G) bodega H) cuarto de preparación de monolitos de suelos, I) laboratorio, J) pasillo lateral y K) sanitario.

La operación del centro puede realizarse a dos niveles:

- Nivel de operación para divulgación. Para esto se requiere, al menos, la contratación de un responsable con grado de doctor, así como del apoyo de tres técnicos con licenciatura: un biólogo, un agrónomo y un geógrafo.

- Nivel de operación como centro generador y divulgador. Se requiere formar un equipo de doctores con las siguientes especialidades: Manejo de bases de datos digitales y Geomorfología; Química analítica y clasificación de suelos; Ecología del paisaje; Percepción remota; Agronomía tropical, Producción animal y Manejo forestal. Cada experto podría ser 
Información técnica para el

desarrollo agropecuario

\section{Cuadro I. EJemplo de DIVULGACIÓN DE LA INFORMACIÓN PARA AGRÓNOMOS}

156
Disponibilidad

Riesgo/ limitación

\section{Evaluación del clima}

Longitud del periodo de crecimiento

Radiación total

Largo del día

Régimen de temperatura

Riesgos climáticos

Tamaño de la temporada de lluvias

Riesgos durante la temporada de lluvias

\section{Evaluación de las geoformas}

Pendiente

Riesgo de erosión

Riesgos de movimiento en masa

Riesgos de colapso

Dificultad de acceso a las formas del terreno

\section{Evaluación del suelo}

Humedad del suelo

Disponibilidad de oxígeno

Disponibiidad de nutrimentos

Capacidad de retención de nutrimentos

Condiciones de enraizamiento

Condiciones que afecten la germinación

Exceso de sales

Exceso de sales de sodio

Toxicidad del suelo (p.e. sat de Al)

\section{Evaluación del manejo de la tierra}

Preparación inicial de la tierra

Trabajo invertido

Potencial para mecanización

Facilidad de acceso actual

Facilidad de acceso potencial

Riesgo de erosión por agua

Riesgo de erosión por viento

Riesgo de inundación

Enfermedades y plagas

\section{Evaluación de la calidad del agua de riego}

Profundidad del acuífero

Salinidad del agua de riego

Sodicidad del agua de riego

Toxicidad por cloro

Toxicidad por boro

Carbonato de sodio residual

Toxicidad por sulfatos

Tecnología para riego

\begin{tabular}{|c|c|c|c|c|}
\hline $\mathrm{ma}$ & $\mathrm{a}$ & $\mathrm{m}$ & $\mathrm{b}$ & $\mathrm{mb}^{*}$ \\
\hline $\mathrm{np}$ & $\mathrm{l}$ & $\mathrm{m}$ & $\mathrm{s}$ & $\mathrm{ms}^{* *}$ \\
\hline
\end{tabular}

\begin{tabular}{|l|l|l|l|l|}
\hline & & & & \\
\hline & & & & \\
\hline & & & & \\
\hline & & & & \\
\hline & & & & \\
\hline & & & & \\
\hline & & & & \\
\hline
\end{tabular}

\begin{tabular}{|l|l|l|l|l|}
\hline & & & & \\
\hline & & & & \\
\hline & & & & \\
\hline & & & & \\
\hline & & & & \\
\hline
\end{tabular}

\begin{tabular}{|l|l|l|l|l|}
\hline & & & & \\
\hline & & & & \\
\hline & & & & \\
\hline & & & & \\
\hline & & & & \\
\hline & & & & \\
\hline & & & & \\
\hline & & & & \\
\hline & & & & \\
\hline
\end{tabular}

\begin{tabular}{|l|l|l|l|l|}
\hline & & & & \\
\hline & & & & \\
\hline & & & & \\
\hline & & & & \\
\hline & & & & \\
\hline & & & & \\
\hline & & & & \\
\hline & & & & \\
\hline & & & & \\
\hline
\end{tabular}

\begin{tabular}{|l|l|l|l|l|}
\hline & & & & \\
\hline & & & & \\
\hline & & & & \\
\hline & & & & \\
\hline & & & & \\
\hline & & & & \\
\hline & & & & \\
\hline
\end{tabular}

$*_{\text {ma }}=$ muy alto; $\mathrm{a}=$ alto; $\mathrm{m}=$ medio; $\mathrm{b}=$ bajo; $\mathrm{mb}=$ muy bajo

** $\mathrm{np}=$ no presente; $\mathrm{l}=$ ligero; $\mathrm{m}=$ medio; $\mathrm{s}=$ severo; $\mathrm{ms}=$ muy severo 
asistido por dos estudiantes de posdoctorado becados por Conacyt. Los objetivos de este grupo de expertos serían: trabajar en la generación de conocimiento para mejorar los sistemas productivos sin deterioro del ambiente; asistencia técnica, por ejemplo, un aumento de $10 \%$ en los volúmenes de producción de cítricos; vínculo entre productores e investigadores de la región; asistencia técnica para los tomadores de decisiones (autoridades de diversos niveles: presidentes municipales, regidores, secretarios de los gobiernos estatal y federal, entre otros).

\section{A manera de conclusión}

En el estado de Yucatán se cuenta con una gran cantidad de información en bases de datos digitales en los ámbitos agrícola, ambiental, social y económico, producto de esfuerzos compartidos entre la Secretaría del Medio Ambiente y Recursos Naturales y la Secretaría de Ecología del Gobierno del Estado de Yucatán, que están promoviendo el ordenamiento ecológico del estado y de la costa yucateca.

Paralelamente, existe un grupo de investigadores apoyados por el Gobierno del estado de Yucatán y el Consejo Nacional de Ciencia y Tecnología, que está desarrollando y evaluando bases de datos digitales del medio físico con el objetivo de zonificar el estado de Yucatán desde el punto de vista agroecológico. Estas actividades son:

a) Análisis y evaluación de las bases de datos digitales (geomorfología, geología, climatología, edafología, hidrología, etcétera)

b) La aplicación de modelos para convertir la información taxonómica (geomorfología, geología, climatología, edafología, hidrología, etc.) en información útil en aspectos agropecuarios y forestales con sentido ecológico para los diversos tomadores de decisiones

c) La integración de la información con objetivos definidos y claros, como en el caso de este proyecto, para la creación de zonas agroecológicas y unidades ambientales

d) La generación de escenarios de acuerdo con el manejo de la información ante eventos futuros esperados o posibles 
Las instituciones e investigadores participantes son: Cinvestav (Dr. Eduardo Batllori); CICY (Dr. Roger Orellana); Uqroo (M. en C. Oscar Frausto y M. en C. Thomas Ihl); UACAM (M. en G. Gerardo Palacio), Colpos (Dr. David Palma) y UADY (Dra. Julia Pacheco, M. en C. Armando Cabrera, Dr. Salvador Flores, Dr. Francisco Bautista), así como asesores de la UNAM, CP, ITC (Holanda), Empresa Brasileña de Investigación Agropecuaria (Embrapa), entre otras.

Por otra parte a nivel nacional se encuentra en consulta pública la propuesta de Ley de Restauración y Conservación de Tierras con la que se pretende impulsar a la agricultura nacional, pero con un enfoque de sustentabilidad. Los diputados y senadores de la República así como otras organizaciones han identificado los grandes problemas del país en los ámbitos ecológico, ambiental, agropecuario $y$ forestal que han impactado de manera fuerte $y$ desfavorable a las sociedades rurales y, en particular, a las indígenas.

Con esta ley se intenta poner orden en las actividades que hacen uso del suelo en zonas no urbanas, se pretende que se haga realidad el pago de servicios ambientales a las comunidades que conserven sus recursos naturales $y$ que exista un padrón de profesionales acreditados para la elaboración de los planes de manejo de las tierras.

Las tres iniciativas mencionadas le dan sustento a la propuesta de formar el Centro de Información Ecológica, Ambiental, Agropecuaria y Forestal del estado de Yucatán. Es deseable que el centro cuente con técnicos del más alto nivel científico y con el grado de doctor, tal y como ocurre en el Departamento de Agricultura de los Estados Unidos (USDA); en el Instituto Nacional de Investigaciones en Caña de Azúcar (INICA) en Cuba, en la Embrapa y en los países europeos.

La idea general es terminar con la larga historia de fracasos de los planes agropecuarios y forestales que han afectado al capital natural y al desarrollo humano de la región.

Nunca más un plan agropecuario sin la suficiente cantidad y calidad de información científica.

\section{FUENTES CONSULTADAS}

Antoine, J., G. Fischer y M. Makowski (1997). “Multiple criteria land use analysis”. Applied Mathematics and Computation, 83(2-3), 195-2I 5. 
Bautista-Zúñiga F., E. Batllori-Sampedro, M.A. Ortiz-Pérez, G. Palacio-Aponte, y M. Castillo-González (2003). "Geoformas, agua y suelo en la Península de Yucatán”, en: P. Colunga y A. Larque (eds.). Naturaleza y sociedad en el área maya. México: Academia Mexicana de Ciencias/ Centro de Investigación Científica de Yucatán.

S. Cram-Heydrich e I. Sommer (2004). "Suelos". en: F. Bautista-Zúñiga y H. Delfín (eds.) Técnicas de muestreo para el estudio del manejo de recursos naturales. México: UNAM/INE/UADY.

Ma. Soledad Díaz-Garrido, J.L. Miguel Castillo-González y Alfred J. Zinck (2005). "Soil heterogeneity in karst zone: Mayan nomenclature, WRB, multivariate analysis and geostatistics". Euroasian Soils Science (en prensa).

H. Estrada-Medina, J. Jiménez-Osornio y J. A. González-lturbe (2004). "Relación entre relieve y suelos en zonas cársticas". Terra Latinoamericana, 22(3), 243-254.

J. García y A. Mizrahi (2005).“Diagnóstico campesino de la situación agrícola en Hocabá,Yucatán”. Terra Latinoamericana, 23(4) (en prensa).

J. Jiménez-Osornio, J. Navarro-Alberto, A. Manu y R. Lozano (2003). "Micro-relieve y color del suelo como propiedades de diagnóstico en Leptosoles cársticos". Terra, 2I, I-I I.

y G. Palacio (2005). Caracterización y manejo de suelos en la Península de Yucatán: implicaciones agropecuarias, forestales y ambientales. México: Universidad Autónoma de Campeche/Universidad Autónoma de Yucatán / Semarnat.

H. Rivas-Solórzano, C. Durán de Bazúa y G. Palacio (1998). “Caracterización y clasificación de suelos con fines productivos en Córdoba, Veracruz, México”. Investigaciones Geográficas, 36, 21 -33.

Carnevali, G., I. Ramírez y J.A. González Iturbe (2003). “Flora y vegetación de la Península de Yucatán”, en: P. Colunga y A. Larqué (eds.). Naturaleza y sociedad en el área maya. Pasado, presente y futuro. México: $\mathrm{CICY} / \mathrm{Aca}-$ demia Mexicana de Ciencias.

González-Iturbe, J. A., I. Olmsted y F.Tun (2002). "Tropical dry forest recovery after long terem henequen (Sisal, Agave fourcroydes) plantations in northern Yucatán”. México Forest Ecology and Management, 167, 67-82. Gregorio, A. y L. Jansen (2000). Land cover classification system. Roma: FAO. 
Larschieid, G.y S. Blackmore (1996). "A precision farming approach to the application of agrochemicals". 3rd. International conference on precision agriculture. Estados Unidos de América: Cranfield University.

Llamas, E., J. B. Castillo, C. Sandoval, F. Bautista Zúñiga (200I). “Trees forage production and quality analysis with forage potentiality on a quarry soil in Mérida, Yucatán, México". International Symposium on Silvopastoral Systems. CATIE-IUfro. San José, Costa Rica. [en línea] Disponible en: http://www.fao.org/WAIRDOCS/LEAD/X6109E/×6109e0a. htm\#TopOfPage

Makowski, M. L. Somlyody y D.Watkins (1996).“Multiple criteria analysis for water quality management in the Nitra basin". Water Resources Bulletin, 32(5), 937-95I.

Olmsted, I., J.A. González-Iturbe, J. Granados, R. Durán y F. Tun (1999). “Vegetación de la Península de Yucatán”, en: P.A. Chico Ponce de León, A. García y J. Córdoba (coords.). Atlas de procesos territoriales de Yucatán. Mérida: Facultad de Arquitectura, UADY.

Orellana, R., M. Balam e I. Bañuelos (1999).’Balance ombrotérmico. Evaluación climática”, en: P.A. Chico Ponce de León,A. García y J. Córdoba (coords.). Atlas de procesos territoriales de Yucatán. Mérida: Facultad de Arquitectura, UADY, 282-293.

C. Espadas y J. A. González-Iturbe (2002). "Aplicaciones de los diagramas ombrotérmicos de Gaussen modificados en la Península de Yucatán”, en:A. Sánchez Crispín (ed.). México en su unidad y diversidad territorial, t. I, Aguascalientes: INEGI, 60-73.

Ortiz, P., et al. (1996). Peligros geomorfológicos de México: mapa escala I:I 000000. Instituto de Geografía, Instituto Nacional de Ecología.

Zinck, J.A. y C. R.Valenzuela (1990). "Soil geographical database: structure and applications examples”. ITC Journal, 3, 270-294 